\title{
Mobile Robotics Activities in DOE Laboratories
}

\section{SPIE Defense \& Security Program}

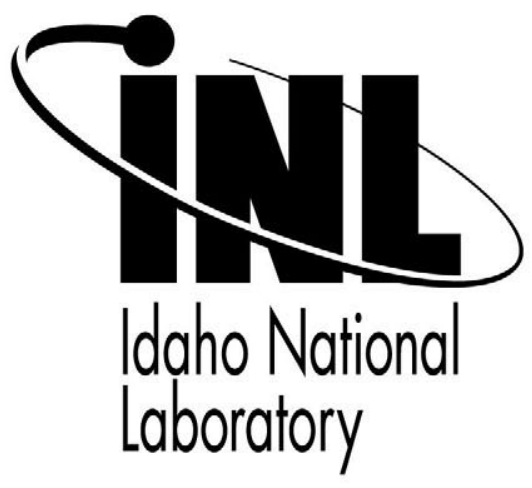

\author{
March 2005
}

This is a preprint of a paper intended for publication in a journal or proceedings. Since changes may not be made before publication, this preprint should not be cited or reproduced without permission of the author. This document was prepared as an account of work sponsored by an agency of the United States Government. Neither the United States Government nor any agency thereof, or any of their employees, makes any warranty, expressed or implied, or assumes any legal liability or responsibility for any third party's use, or the results of such use, of any information, apparatus, product or process disclosed in this report, or represents that its use by such third party would not infringe privately owned rights. The views expressed in this paper are not necessarily those of the United States Government or the sponsoring agency. 


\title{
Mobile Robotics Activities in DOE Laboratories
}

\author{
Ron Lujan ${ }^{\mathrm{a}}$, Jerry Harbour ${ }^{\mathrm{a}}$, John Feddema ${ }^{\mathrm{b}}$, Sharon Bailey ${ }^{\mathrm{c}}$, Jacob Barhen ${ }^{\mathrm{d}}$, David Reister ${ }^{\mathrm{d}}$ \\ ${ }^{a}$ Idaho National Laboratory, PO Box 1625, Idaho Falls, ID 83415 \\ ${ }^{\mathrm{b}}$ Sandia National Laboratories, PO Box 5800, Albuquerque, NM 87185-1003 \\ ${ }^{\text {c}}$ Pacific Northwest National Laboratory, PO Box 999, Richland, WA 99352 \\ ${ }^{\mathrm{d}}$ Center for Engineering Science Advanced Research, Oak Ridge National Laboratory, Oak Ridge \\ TN 37831-6016
}

\begin{abstract}
This paper will briefly outline major activities in Department of Energy (DOE) Laboratories focused on mobile platforms, both Unmanned Ground Vehicles (UGV's) as well as Unmanned Air Vehicles (UAV's). The activities will be discussed in the context of the science and technology construct used by the DOE Technology Roadmap for Robotics and Intelligent Machines (RIM) ${ }^{1}$ published in 1998; namely, Perception, Reasoning, Action, and Integration. The activities to be discussed span from research and development to deployment in field operations. The activities support customers in other agencies. The discussion of "perception" will include hyperspectral sensors, complex patterns discrimination, multisensor fusion and advances in LADAR technologies, including real-world perception. "Reasoning" activities to be covered include cooperative controls, distributed systems, ad-hoc networks, platform-centric intelligence, and adaptable communications. The paper will discuss "action" activities such as advanced mobility and various air and ground platforms. In the RIM construct, "integration" includes the Human-Machine Integration. Accordingly the paper will discuss adjustable autonomy and the collaboration of operator(s) with distributed UGV's and UAV's. Integration also refers to the applications of these technologies into systems to perform operations such as perimeter surveillance, large-area monitoring and reconnaissance. Unique facilities and test beds for advanced mobile systems will be described. Given that this paper is an overview, rather than delve into specific detail in these activities, other more exhaustive references and sources will be cited extensively.
\end{abstract}

Keywords: UGV, UAV, autonomy, mobility, HMI, sensors, surveillance, monitoring, security,

\section{INTRODUCTION}

The Department of Energy sites and laboratories have been involved with robotics and remote systems for decades, primarily to reduce the exposure to personnel involved with hazardous operations. In the 1980's the activities evolved to mobile platforms in the form of teleoperated robots to meet the needs in operation of DOE nuclear facilities. Eventually, the laboratories began to push the state-of-the art in mobile platforms in the 1990's to include more sensor fusion and advanced controls, specifically cooperative behavior of air and ground platforms. In 1998, the laboratories conducted a technology roadmap to create a vision of Robotics and Intelligent Machines (RIM). In that roadmap, a construct was developed to structure the discussion on the science and technologies needed to meet future needs. The construct of sciences and technologies was based on the human behavior model (Sensing, Reasoning, and Action) with an additional element, Integration. The roadmap was also focused on the ultimate objectives driving the new technologies, i.e., reduce personnel exposure, reduce impact to the environment, increase productivity (decrease cost, increase production rate, increase quality, increase accuracy, etc.), decrease programmatic/project risk, etc. The mobile vehicle activities going on in four of the DOE national laboratories (Idaho National Laboratory, Sandia National Laboratories, Pacific Northwest National Laboratory, and Oak Ridge National Laboratory) will be discussed in terms of the elements of the RIM construct and how they contribute to achieve the ultimate objectives for customers. The activities in the laboratories extend beyond DOE customers to other agencies and the private sector. 


\section{IDAHO NATIONAL LABORATORY (INL)}

The Idaho National Laboratory has been developing and deploying unmanned ground vehicles (UGV's) since the early 90's. INL activities in unmanned air vehicles (UAV's) began in the late 90's building on it's autonomous behaviors developed for UGV's. These activities have been funded from a variety of sources including internal R\&D funds, operations funding from INL facility customers, and Work for Others customers in government agencies and the private sector. In many cases, building realistic customer expectations and trust in robotic systems has a maturation process involving "baby steps". This philosophy not only entails establishing an understanding or vision of the end product with the customer in terms of user requirements. It also involves working toward interim realistic goals based on the customer/user's resource limitations, acceptance of risk and new technologies, and a pragmatic understanding of the state-of-the art. The systems often begin at rudimentary levels to work within the customer's comfort zone, but evolve in greater integration as system trust and expectations rise. The following projects illustrate some of the mobile vehicle efforts that are ongoing at the INL utilizing that philosophy.

Radiation and Photographic Survey Vehicles: The Idaho Completion Project Environmental Services Program is responsible for performing radiation and photographic surveys at the 890 -square mile, high desert Idaho National Laboratory site to assess the impacts of operations on the environment. Three distinct types of platforms have been developed and deployed for the customer. They are the Remote All-Terrain Vehicle or RATV (wheeled UGV), the Remote Air Monitoring System (RAMS) (fixed wing UAV), and the Mini-heli-cam (rotary wing UAV). All these systems are currently in use by that customer.

The Remote All Terrain Vehicle (RATV) ${ }^{2}$ replaces a manually-driven HUMVEE and pickups with two-person teams required on board each for safety, navigation, and data-gathering during field radiation surveys. The RATV platform is a commercially-available amphibious Trident Predator all terrain vehicle (See Figure 1). It has been modified to allow manned, remote, or fully autonomous operational modes. The latter two only require one operator in an atmospherically controlled trailer. A scintillation radiation detector and a high-resolution gamma spectrometer are mounted on the platform. The RATV's minimum speed of $3 \mathrm{mph}$ allows for more accurate readings from the rad detector than was possible on the HUMVEE. Autonomous parallel path swathing developed at the INL to minimize "skip" and "overlap" has been employed. A spatial mapping program provides immediate reporting of survey results. When elevated levels are indicated, the gamma spectrometer can be immediately employed to evaluate specific isotope concentrations in surface soils. Subsequent measurements can be repeated and evaluated very accurately over a period of years. At the Test Reactor Area alone, there are 200 such locations. The amphibious design presents no fire hazards and can be operated when fire restrictions are applied off-road on the site during dry seasons. Deployments of the RATV have demonstrated an $87 \%$ reduction in labor costs, reduced risk of exposure to personnel, more accurate and complete results, and an ability to survey in contaminated and hazardous environments that were previously restricted. Contingent on funding, future plans include integrating obstacle detection sensors developed for the DARPA PerceptOR program and other programs.

In addition to ground based air monitoring systems, the customer required airborne monitoring of plumes, especially in the case of wildfires, which are not uncommon in the area. In the case of wildfires, people are restricted to remain 2 to 5 miles away from the fire. The Remote Air Monitoring System (RAMS) ${ }^{3}$ (See Figure 2) was developed to provide real-time monitoring of the plumes from wildfires safely at low cost. The

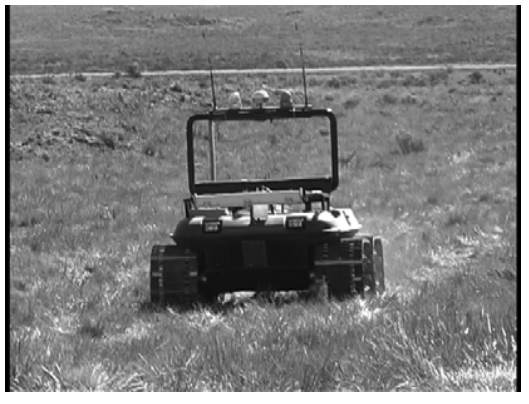
platform is a commercially-available, fixed-wing Rascal $110 \mathrm{R} / \mathrm{C}$ airframe capable of flight in winds exceeding $25 \mathrm{mph}$. It has been modified to include GPS-based autonomous operations, a 15-mile

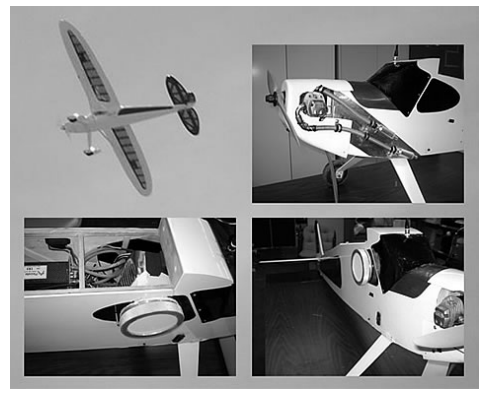


operational radius, increased payload/lift capacity, and increased flight duration (1 hour). A hand-held High Altitude Air Sensor (HAAS) has been modified and integrated into the platform. The battery was changed from NiMH to Li-Poly providing greater capacity at one half the weight. New higher-efficiency motor and impeller were utilized. These changes provide ten times the sampling flow rate over a 30-minute duration. The system was successfully tested in 2004. RAMS provides a new capability not previously available to the customer. Contingent on funding, future plans include testing with a controlled burn and collecting samples from the smoke plume.

The customer also required aerial photography for various applications, including state regulatory permits, site security, and wildfire monitoring. The previous method involved hiring a private plane and pilot and obtaining special permits. The Mini-heli-cam was developed to provide an alternate solution ${ }^{3}$. It is a low-cost, commercially-available Thunder Tiger Raptor 50 (See Figure 3) which is an $\mathrm{R} / \mathrm{C}$ rotary-winged platform with a rotor diameter of 53-5/8 inches. It has a 8.5 pound payload capacity and a ceiling of 1000 feet above ground level (agl). A small still camera with a capacity of 248 4-megapixel images has been mounted on the platform and can be operated from a common ground receiver package with video recording. The platform and ground receiver package are highly transportable. The Mini-heli-cam has been successfully deployed and has demonstrated a $70 \%$ reduction in operating costs utilizing existing personnel with no lead time required for contracting and permitting.

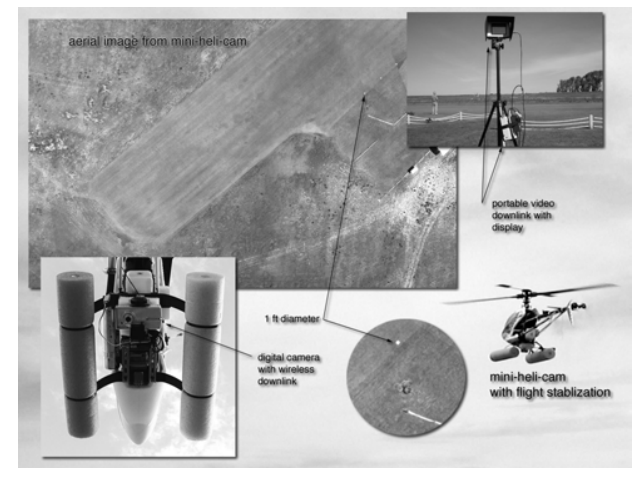

Sensor Characterization Test Vehicle: A customer at the BechtelNevada Remote Sensing Laboratory (RSL)-National Center for Counter Terrorism (NCCT) conducts intrusion security sensor (rad, motion, heat, machine vision, etc.) characterization tests. To conduct tests, the customer formerly used a dolly with a mannequin as a target. Operators simulated motion by pulling ropes attached to the dolly using tape on the floor to designate intended paths. The work was slow, tedious, inaccurate, and not repeatable. The customer required increased path accuracy $(+/-0.5 \mathrm{in})$, an ability to change path patterns easily, and platform speeds from a crawl to a sprint. The customer required that mannequin conditions such as temperature be controlled and monitored remotely. The customer agreed to interim goals while progress in the state-ofthe-art and subsequent additional funding made final goals more feasible. To meet these goals, the INL developed a system is based on a modified PowerBot commercially available from ActiveMedia ${ }^{4}$. Route specification was achieved with a tool called the INL DXF Editing Application (IDEA) created to convert Drawing Exchange Format (DXF) Files created by the operator into lists of waypoints. Accurate positioning and path following without GPS in the RSL facility was achieved by incorporating a version of the Markov localization method and by representing the robot's state space based on Monte Carlo sampling. This provided an extremely efficient technique for mobile robot localization. Overall automation was achieved within LabVIEW, a commercially available application used by RSL, by using an ActiveX control that mediates between a data acquisition system, the Robot and the INL graphical interface. The system was delivered to RSL in June 2004 for acceptance testing and was deployed for operations in November 2004 (See Figure 4). RSL reports that 3 weeks of sensor characterization testing using previous methods was accomplished in one day. A path accuracy of $+/-5 \mathrm{~cm}$ accuracy was attained at $1 \mathrm{~m} / \mathrm{sec}$ which exceeds previous capabilities and meets customer interim goals. The paths were highly repeatable. Future plans, contingent on funding, include further refinements to the system to increase accuracy.

Simultaneous Multi-Platform Autonomous Flight: The DARPA Future Combat Systems Communications ${ }^{5}$ program focus was on wireless ad-hoc mobile networking with anti-jamming and low probability detection. The INL role was to provide a low-cost FCS targeted UAV solution for Demo 3 Go/No-Go activities with responsibilities for air frame and communications payload integration, launch and recovery operations. The demonstration involved a single pilot to launch and recover each vehicle while a single command and control ground operator and RF telemetry laptop link 
provided health status monitoring and course alteration. A record was established when 5 UAV's were flown autonomously within the same two-kilometer airspace from a single ground station.

Unmanned Protection Systems: Unmanned vehicles are primarily fielded as single assets to perform restricted, albeit critical mission functions. It is envisioned that multiple unmanned vehicles will be deployed to complete more complex mission scenarios. The objective of an INL project planned for FY-05/06 and funded by the Joint Robotics Program (JRP), entitled Enabling Technologies for Unmanned Protection Systems, is to integrate and mature existing base unmanned vehicle-related technologies and conduct relevant field experiments to quantitatively assess mission efficacy ${ }^{6}$. The project will leverage heavily upon ongoing UAV and UGV work at the INL as well as other JRP programs. The template for experiments will include UAV detection of potential target of interest; cueing of UGV's or other unmanned assets to target of interest; on-ground evaluation and assessment; and execution of response tasks. The following technologies will be applied:

- Time sensitive Delivery of Geo-registered spatial data

- High resolution still imagery

- Collaborative Search and detection behaviors

- Countermine detection, marking and neutralization

- Real-time EO/IR ident, fusion, and monitoring

- Common Op. Control Unit with scalable representation

- Indoor/outdoor synchronized positioning

- Augmented virtuality interactive interface

- Real-time SLAM

- Human Presence Detection

- Geo-referenced mosaic

- Stabilization and tracking

- Probability-based vectored response

- Adaptive coverage behavior

- Unmanned vehicle collaborative patrol

UAV Test Range: The INL has constructed a UAV Test Range on which to conduct testing of UAV technologies at a site located 45 miles from the Idaho Falls airport at about 5000 feet above sea level. It has a 1000' by 100' paved runway. There are isolated controlled access boundaries for testing. The INL possesses communications spectrum authority according to the National Telecommunications and Information Agency (NTIA) Manual of Regulations and Procedures for Federal Radio Frequency Management, the "NTIA Redbook". In addition, the INL possesses an FAAapproved Unmanned Aerial Vehicle "Certificate of Authorization". These capabilities provide the INL with the ability to develop, test, and validate unmanned platforms in singular and integrated system manner.

Human-Robot Testing and Guidelines: INL's experience in developing field deployable mobile vehicles as described above made it very evident of the need for human-robot system testing. True human-robot teaming requires that all members are aware of the goal, and can work toward that goal with or without input from the other members. For the control architecture of a robotic system to be useful and usable, it must allow the human team member to build trust in the system, regardless of the intelligence inherent in the robotic system. System trust can only be enhanced if the system is designed to meet actual users' needs, abilities, and limitations within the constraints of the task; such design requires true user testing, not simple designer evaluation. To understand these challenges and funded by Laboratory Directed Research and Development, INL personnel conducted human factors testing on a variable autonomy control architecture using a simplified search and rescue scenario with a complex, real-world environment ${ }^{7}$. The testing involved an $i$ Robot ATRV Jr. with a full range of sensors. The sensory information was fused to support an environmental on-the-fly mapping program. Aspects studied included the operator's environment; teaming interaction with autonomy; human abilities, expectations, limitations; and the dynamic nature of the task. The testing provided extensive lessons learned that are provided in the paper. From these, specific detailed guidelines were developed for human-robot testing as summarized below:

- Test in real-world conditions; not simplified

- System design must be based on how human will work with system

- Test environment must reflect complexities of real world

- Test environment must incorporate uncertainties of environment

- Robot's capabilities must be designed to exploit environment
- Involve users in testing who are familiar with application

- Incorporate need for operator to be aware in more than one environment.

- Test must address teaming and human trust of robot

- Tasks constraints may change with teaming; but may still shape human expectation of system behavior. 


\section{SANDIA NATIONAL LABORATORIES}

Sandia National Laboratories Intelligent Systems and Robotics Center has been developing mobile robotic platforms since the mid-1980s. Department of Energy (DOE) applications for mobile robotics include security, environmental remediation, materials storage and monitoring, and response and cleanup of accidents involving nuclear materials. In addition to DOE activities, Sandia has developed several mobile robotic platforms for other defense agencies including the DoD Joint Program Office, Special Operations Command, Marine Corps Warfighting Laboratory, and DARPA. Below is a very brief description of only a few of these systems. More information on these and other robotic systems can be found in the referenced background paper ${ }^{8}$.

Accident Response UGV's: The Accident Response Mobile Manipulator System (ARMMS) in Figure 5(left) was developed for DOE accident response. Combining a Humvee (HMMWV) as a basic mobility platform and two Shilling manipulators, ARMMS was developed with the full range of capabilities necessary to provide a comprehensive response for nuclear accidents. It can be deployed in a teleoperational capacity using a single fiber optic cable that provides ARMMS a range of up to four kilometers, or remotely via a radio frequency (RF) communication link. In the event of damage to the fiber optic cable, the RF system is automatically enabled for vehicle movement or retrieval. ARMMS can be driven like a regular Humvee and carries an equipment shelter that can be deployed as a command post for teleoperated activities. Coordinated Cartesian-based control of the Shilling manipulators is provided by the Sandiadeveloped SMART (Sandia's Modular Architecture for Robotics and Teleoperation) control software 9 . This same control architecture has been used to control several other mobile manipulators such as the Remotec Platform shown in Figure 5 (right).

An example of a smaller Sandia-designed robot is Atlas (see Figure 6), a longer-range mobile robot platform based on a dual-articulated body design. The vehicle's design is based on the previously developed SandDragon, which was a manportable ground robot developed for the Marine Corps Warfighting Lab (MCWL) as part of the Forward Attack Networked Ground System (FANGS) project. The Atlas vehicle is approximately 6 feet long and 3 feet wide. Between two equal-sized tracked bodies is a pitch/roll joint that maximizes ground contact and improves mobility over rugged terrain. The system is powered by 25 Lithium-Ion batteries containing approximately $3.7 \mathrm{KW}-\mathrm{Hrs}$ of energy. Expected round trip range is two miles or more, depending on terrain conditions. The system uses a single digital radio transmitting compressed video, commands, and status at about $50 \mathrm{Kbits} / \mathrm{sec}$, with approximately $40 \mathrm{Kbits} / \mathrm{sec}$ dedicated to video. A repeater radio is flown on a helikite to maximize line of sight and extend communications range. Atlas can accept a variety of payloads, such as robot manipulators, mounted on either body, and it runs on the same SMART software as the previously mentioned vehicles.
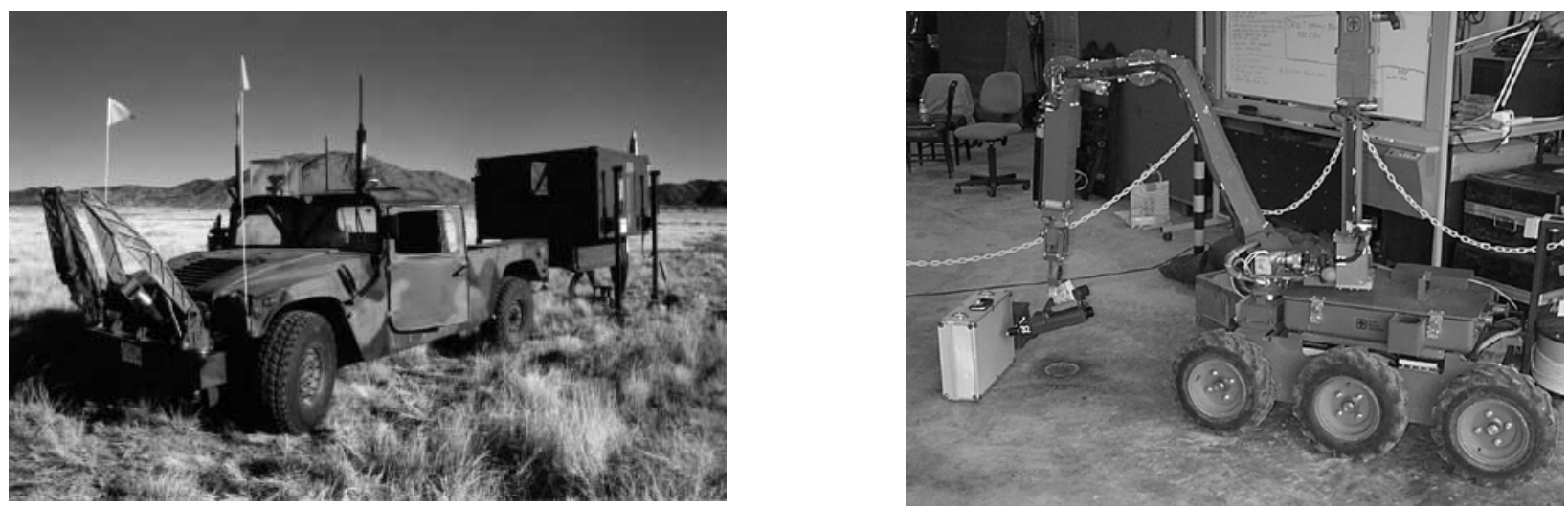

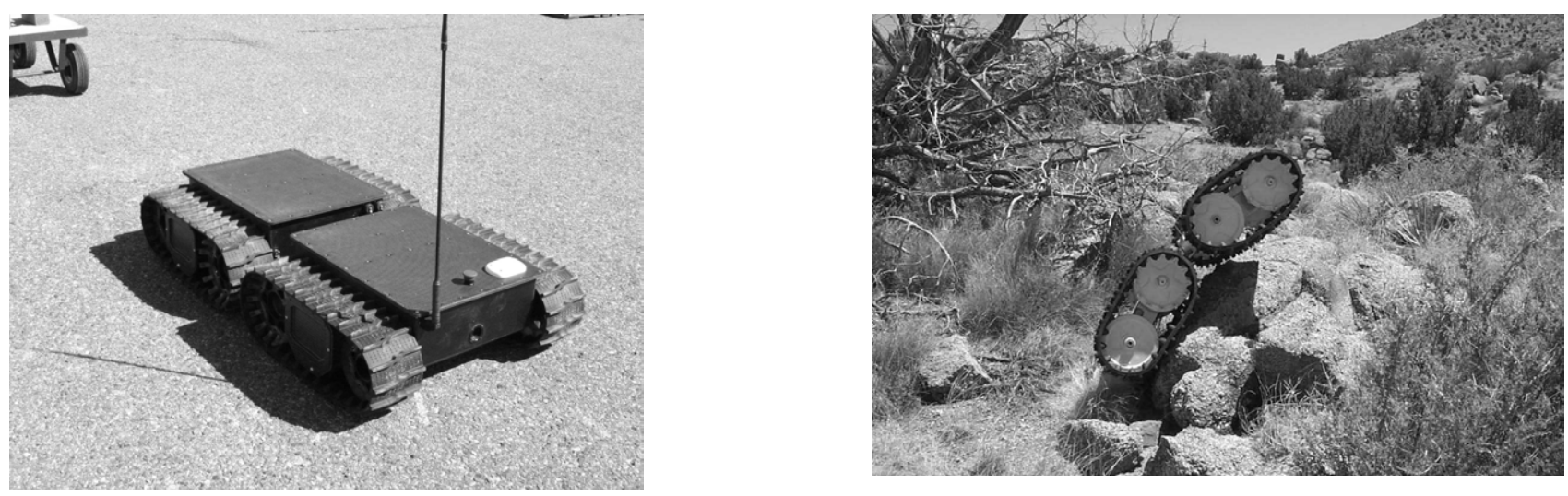

Area Denial and Surveillance-Hopping Robots: Sandia has also developed even smaller mobile platforms for area denial and surveillance missions. For example, Sandia National Laboratories has developed the hopping robots in Figure 7 as part of DARPA Self-Healing Minefield program. The objective of this project was to develop cooperative hopping landmines that can heal breaches and reconfigure upon command. The landmines were designed to be selfcontained and not rely on GPS. As a collective, they form a local ad hoc network, determine their relative position to each other, decide when a neighboring landmine has been removed, and move to the position of missing landmines.
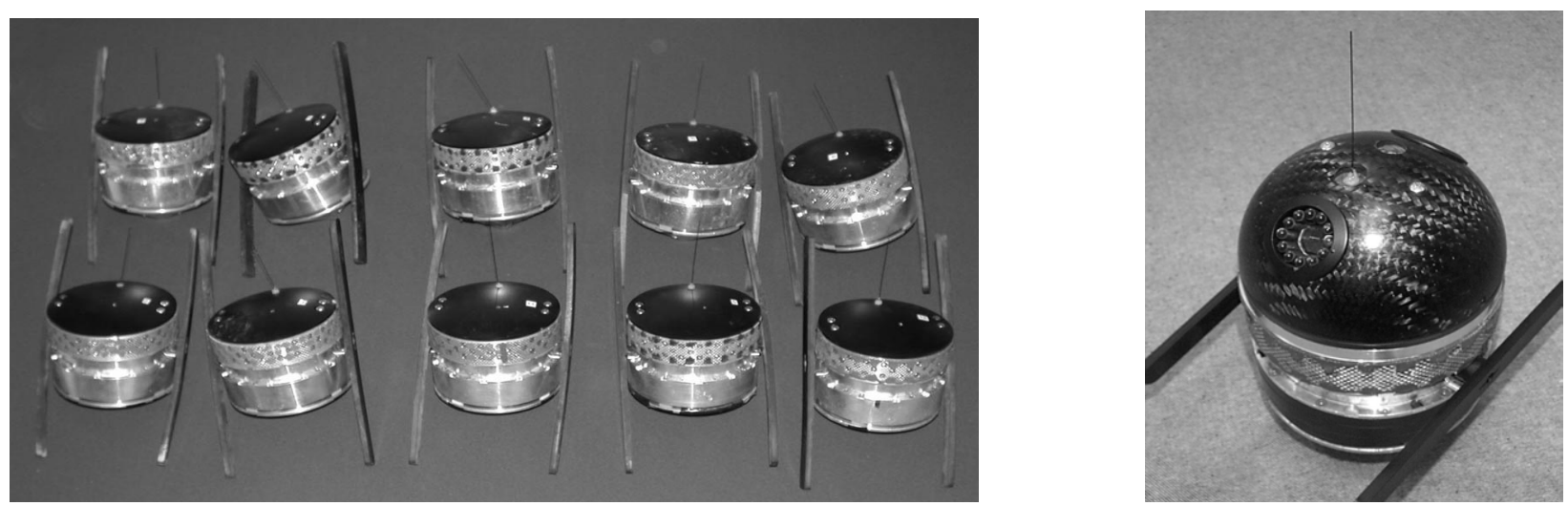

These same hopping robots have been used in a cave reconnaissance mission where each unit was outfitted with two CMOS cameras and IR illuminators. Using the acoustic ranging system to determine the distance between each unit, the hopping robots autonomously spread out inside the cave, forming a communication network where images from deep within the cave are relayed back to the cave entrance. These hopping robots on the left are approximately $12 \mathrm{~cm}$ diameter by $11 \mathrm{~cm}$ tall, and they are able to perform 100 hops, averaging 3 meters high by 3 meters long. They weigh $1.82 \mathrm{~kg}$ of which $0.8 \mathrm{~kg}$ is payload for an explosive charge. The two struts are used to self-right and steer the robots with feedback from a 3-axis accelerometer and magnetometer. Relative positions between hopping robots are determined using a combined RF/acoustic ranging system. The hopping robot on the right is equipped with CMOS cameras and IR illuminators used in cave reconnaissance missions. 
The latest Sandia hopping vehicle, the Wheeled Hopping Vehicle (WHV), combines the hopping actuation with an ordinary wheeled vehicle and shock mitigation. This hopping capability allows a small wheeled vehicle to overcome obstacles over seventeen times its own height. The WHV (See Figure 8) is $30 \mathrm{~cm}$ long x $30 \mathrm{~cm}$ wide $\times 14 \mathrm{~cm}$ tall, weighs $1.6 \mathrm{~kg}$. It is based on a linear-piston, combustion-powered actuator. The actuator servo can rotate the propulsion actuator in either direction, so the WHV is invertible, allowing it to be driven upside down. The WHV unit carries enough fuel to perform approximately 50 hops with a maximum hop height of 2.5 meters (8.2 feet) and a maximum distance of approximately 4 meters $(13.1$ feet). The skid steering system provides directional control for each hop by simply orientating the front of the vehicle towards the required direction of the hop. Currently, the vehicle travels at 8 meters/minute and has a range of $1.4 \mathrm{~km}$.

Cooperative Controls: In addition to designing, building, and testing robotic platforms, Sandia has considerable experience in developing distributed cooperative controls for mobile robotics. Sandia has developed and demonstrated decentralized control algorithms for

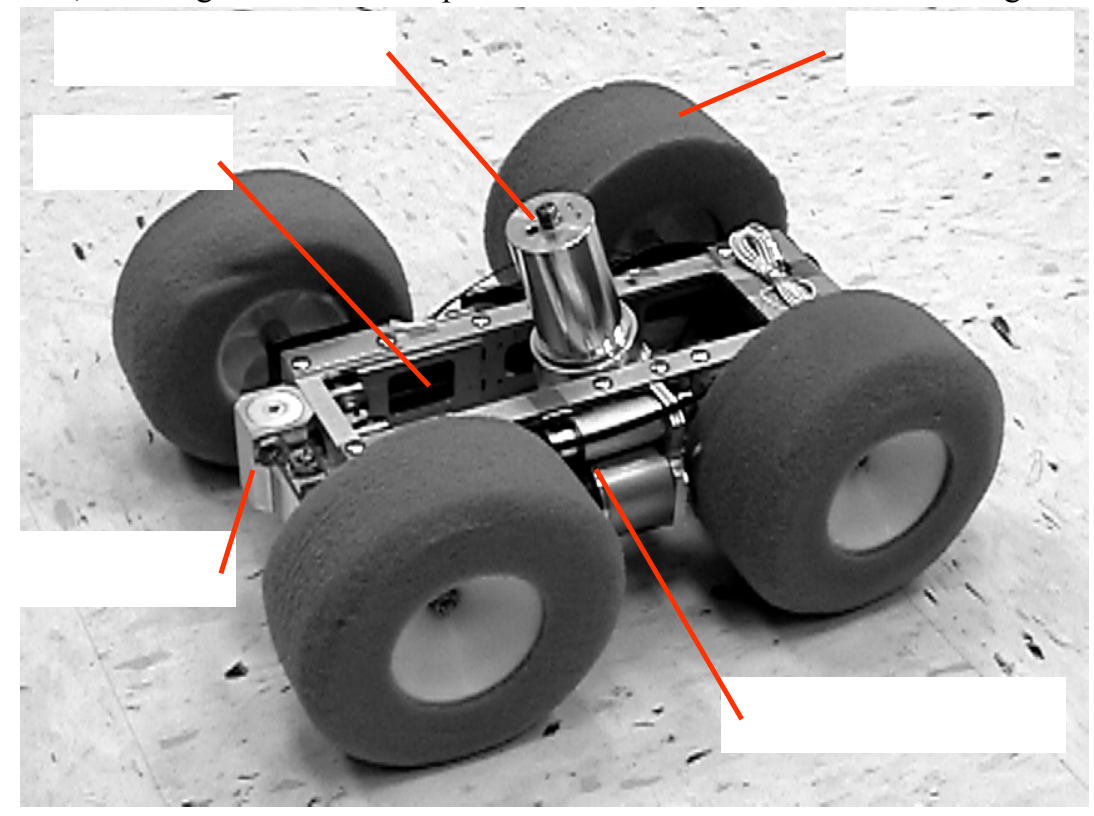
formation following, perimeter surveillance, facility surround, building search, minefield reconfiguration and healing, and chemical plume localization missions ${ }^{10,11}$. These distributed control algorithms were developed in the following DARPA Programs: Tactical Mobile Robots (TMR), Distributed Robotics, Software for Distributed Robotics (SDR), Mobile Autonomous Robots Software (MARS), and Self-Healing Minefield.

\section{PACIFIC NORTHWEST NATIONAL LABORATORY (PNNL)}

PNNL activities discussed below have been funded by internal R\&D funds and DARPA.

GOAT (Goes over all Terrain) Vehicle: The GOAT vehicle is an outgrowth of the DARPA UGCV1 ${ }^{12}$ program where a much larger vehicle was designed as a field reconnaissance vehicle. A goal of that program was to produce a scaleable, modular design, which led to the design the GOAT vehicle shown in Figure 8. The unique capabilities of GOAT come from the novel configuration, which puts the drive wheels on the ends of actuated arms. This is similar to the Sample Return Robot (SRR) at JPL which has the capability to adjust the angle between its shoulder strut angles ${ }^{13}$. GOAT on the other hand uses individually powered shoulder joints, allowing the angle of each shoulder joint to be set independently with respect to the body. As with the SRR, GOAT's shoulder configuration allows it to change body height and posture. However, GOAT's shoulder configuration allows greater control of posture and reduces the reactive forces at the shoulder joint.

The GOAT is targeted to be an effective tool for users operating in EOD, terrorist, and physical disaster situations in urban settings. The vehicle is 30 inches wide, 24-36 inches long, and 24 inches in height. This size allows for access to all normal halls, doorways, stairs and elevators. The footprint can vary from slightly over 12 inches to approximately 40 inches, with a normal operating length of 28 to 30 inches. The wide range of wheelbase allows for stances that range from low crawl to vertical body orientation for maximum look-over capability. The operational flexibility of GOAT transcends those of normal wheeled or tracked vehicles by incorporating individually articulated swing arms. During most operation, the vehicle moves with the efficiency of a normal wheeled vehicle, consuming $1 / 2$ the energy required for a tracked vehicle. However, when faced with curbs or stairs, GOAT can change the body attitude and arm 
orientation to allow for efficient climbing, a feat that other wheeled vehicles cannot achieve. It can move slowly and quietly or speed up to 9 feet per second when required. With a weight of 200 pounds, and a modular design, GOAT can be quickly assembled from components in transportable shipping cases in minutes and configured for the task at hand. The vehicle is designed to accept manipulators, sensors and other packages through its modular design.

The frame is an extremely strong welded steel construction, monocoque design. Interior components are resiliently mounted to the interior space frame. Identical, modular swing arms are mounted on the side of the chassis in a staggered orientation that offsets them both forward and aft of center as well as above and below a centerline. Each shoulder attachment point corresponds to an interior mechanism that functions as both a suspension system and a swing arm repositioning system. Thus, any wheelswing arm can be independently positioned to provide optimal maneuverability. This capability provides the ability to alter road clearance dynamically, as well as change the body attitude to the ground. Additionally, it offers a variable wheelbase as well as the ability to raise and lower individual swing arms to

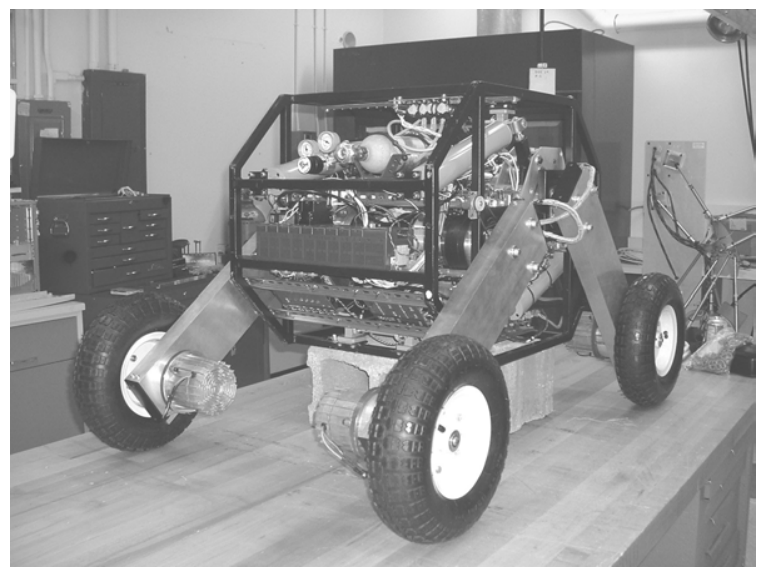
cope with climbing and obstacle negotiation. Should it be tipped over, the swing arms can be used for inverted operation or vehicle self-righting. Each wheel is motor driven, providing maximum traction. The modular design allows other swing arm and wheel/tire combinations to be adapted as required for specific tactical situations. Contingent on funding, future plans include further enhancements to the system to improve motor control and suspension.

Levels of Autonomy: The goal of the Levels of Autonomy research project ${ }^{14}$ is to create a new type of control architecture in which the operator and robot share control. The robot can be as autonomous as the operator believes suitable for the current situation. When the robot cannot handle a situation, more control is given to the operator. This allows the operator to focus attention elsewhere and even to control multiple robots. While the project looked at applying Levels of Autonomy to a reconnaissance mission, it is broadly applicable to any humanrobot system.

The Levels of Autonomy architecture has five distinct levels of human-robot control sharing: Mapping Autonomy, Supportive Autonomy, Safeguarded Autonomy, Waypoint Autonomy and Decision Autonomy. In mapping autonomy the operator controls

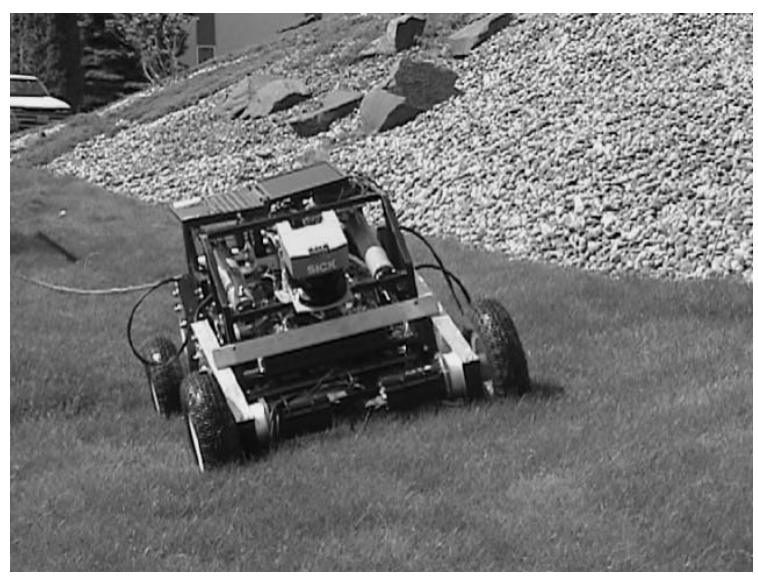
all actions and the robot passively creates maps to help the operator understand the environment and the robot's location. In supportive autonomy the robot suggests actions based on its local knowledge. Safeguarded autonomy permits the robot to override the operator's actions if they will damage the robot. Waypoint autonomy allows the operator to give a series of destinations which the robot drives to autonomously. Finally, decision autonomy gives the robot full control of all decisions (the operator can always change the autonomy level to maintain control).

The Levels of Autonomy control architecture was demonstrated on the GOAT vehicle in outdoor terrain, testing the Mapping, Safeguarded and Waypoint Autonomy modes while exploring and mapping out a region of interest. See Figure 9. The next steps to perform in this work are to demonstrate the usefulness of Levels of Autonomy over traditional teleoperation in a variety of remote missions. Building on the success of reconnaissance missions, this technique could be demonstrated in search and rescue, intruder detection and with the addition of appropriate radiation or chemical sensors to map out the extent of contamination after an accident or attack. 


\subsection{Oak Ridge National Laboratory (ORNL)}

Established by DOE/BES in 1983, the ORNL Center for Engineering Science Advanced Research (CESAR) conducts fundamental theoretical, experimental, and computational research to develop enabling science and technology for intelligent systems. Currently, its primary activities are in support of DOD and the Intelligence Community. Examples of research topics include: mobile cooperating robots, multi-sensor and computer networks, missile defense $\left(\mathrm{BMC}^{3}\right.$, war games, HALO-2 project, and multi-sensor fusion), sensitivity and uncertainty analysis of complex models, global optimization, laser array synchronization (directed energy weapons, LADAR), Digital Signal Processors (DSPs) with digital optical core (16 TeraOPS per chip), quantum optics applied to cryptography, and nanoscale science.

CESAR has achieved world-class recognition, as substantiated by a distinguished publication record, numerous patents and awards (including 3 R\&D 100 Awards), and sustained collaborations with leading research institutions worldwide. CESAR sponsors include: MDA, DARPA, ONR, NASA, NSF, NSA, ARDA, NRO, DOE/SC, $\mathrm{DOE} / \mathrm{FE}$, Army, OSD/JTO, and private industry.

Petascale Computing Systems: CESAR is collaborating with Lenslet Labs (Israel) and Weizmann Institute of Science (Israel) to apply the EnLight processor, which is a DSP with an embedded optical core and over 16 TeraOPS per chip (Figure 10). Matrix

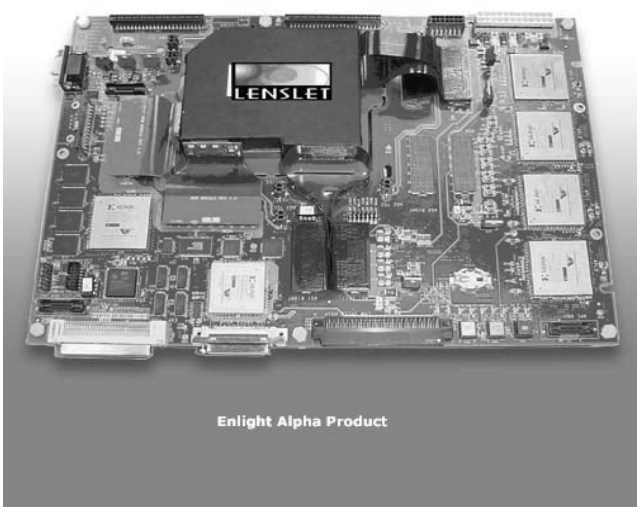

Figure 0. Enlight Petascale Processor vector multiplication is at the heart of many scientific and defense applications that could be leveraged for mobile platforms. In bioinformatics these systems aid to correlate gene data and genetic profiles and to multiply matrices allowing biotech calculations to be greatly accelerated. They have the ability to perform very long Fourier transforms. Therefore these powerful correlation engines will improve detection and extraction of image and audio features as well as and other parameters like behavioral analysis, for reliable automated screening of massive amount of data and for identifying potential threats no matter how rare may they may be.

Advances in Cooperative Control: Supported by DOE and DOD, CESAR has developed the ALLIANCE architecture, which is a novel distributed control technique. ALLIANCE is the first architecture proven to enable autonomous action selection, even in midst of: robot failures, sensor/actuator uncertainties, dynamic environment, and mission changes. It is the first architecture demonstrated on a variety of cooperative teams of heterogeneous robots (Figure 11).

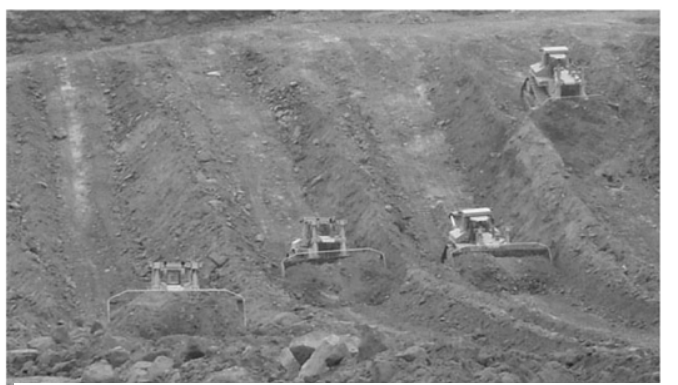

Figure 0. ALLIANCE Autonomous Team

Advances in LADAR Technology: CESAR has developed fundamental technology that successfully addresses the main challenges facing LADAR systems in terms of improved capabilities for target acquisition, discrimination, and interception. A compact high-power light source for LADAR transmitter based on a synchronized broad-area semiconductor laser array has been developed (Figure 12). It has much higher efficiency than solid-state lasers and requires considerably less on-board power.

CESAR has developed an ultra-sensitive coherent detection system for LADAR receiver that can sense an extremely weak optical signal and achieve a high SNR level. The

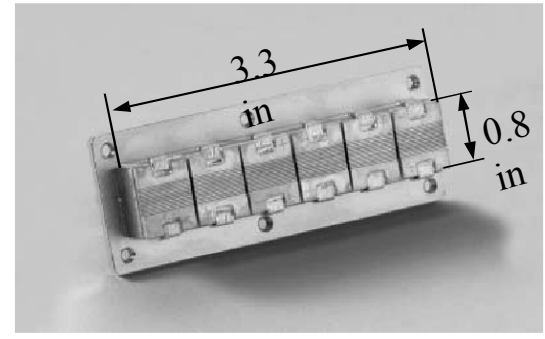

Figure 0. A stacked Array with 3.9 kW output power. technology has unique capabilities. The LADAR transmitter has an average power in excess of 3KW (single mode). 
The receiver is based on nonlinearly amplified regenerative optics that increases detection sensitivity at least by a factor of 1000, and improves the SNR level by at least $30 \mathrm{~dB}$ beyond state of the art in optical detection technology. Novel algorithms have been designed for nonlinear target discrimination and high precision ranging using adaptive laser pulse trains.

Hyperspectral Perception: Hyperspectral imaging sensors map environment in hundreds of different spectral bands in both the visible and invisible wavelengths and dramatically increase the perception capabilities of RIMs. Image analysis can detecting features invisible to conventional computer vision systems. Supported by DOE, CESAR has developed new classification algorithms for hyperspectral data. Geometrical maps in which each pixel has a color that is determined by its spectral class provide a vivid display of feature locations (Figure 13).

For cooperating UAV and UGV, UAV hyperspectral sensing can be integrated with other sensors on UGV to prove a complete picture of the current state of the environment. Maps provided by the analysis of the UAV data can provide targets for further investigation by the UGV.

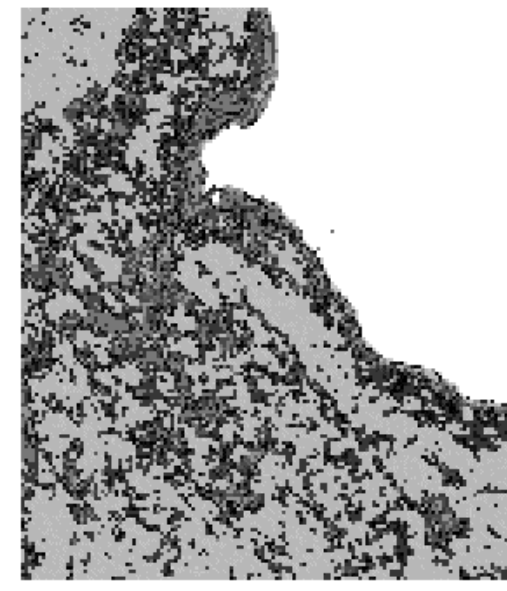

Unconventional Sensing Technology: CESAR has developed new methods for complex patterns discrimination. New methods have been developed for tracking complex targets whose shape evolves in time and for covertly detecting acoustic signals using synchronized laser microarrays.

CESAR is a leader in developing new algorithms for multisensor fusion. Algorithms have been developed for partially known sensor distributions. Mathematical proofs demonstrate that fusion is better than the best sensor or the best subset of sensors.

\section{REFERENCES}

1. "Robotics and Intelligent Machines in the U.S. Department of Energy, A Critical Technology Roadmap", October 1998, Sandia Report SAND98-2401/2. http://www.rim.doe.gov/ .

2. Mark A. Verdoorn, Matthew O. Anderson, Mark D. McKay, Dennis W. McBride, Christopher P. Oertel, "Development of Autonomous Unmanned Vehicles for Large Area Radiation Surveys and Sampling", $10^{\text {th }}$ Robotics and Remote Systems Mtg. Proceedings, pp. 63-68, ANS, Gainesville, Florida, March 28-31, 2004.

3. INEEL News Release, Debra Kahl, "INEEL Researchers Find New Uses for Unmanned Aerial Vehicles," dated TBD.

4. David Bruemmer, Douglas Few, Miles Walton, Heather Hunting, Simon Sirin, “Autonomous Robot System for Sensor Characterization”, $10^{\text {th }}$ Robotics and Remote Systems Mtg. Proceedings, pp. 270-275, ANS, Gainesville, Florida, March 28-31, 2004.

5. INEEL News Release, Kathy Gatens and Scott Bauer, "Simultaneous Flight of Five Autonomous Unmanned Aerial Vehicles (UAV's) Makes History," dated August 7, 2003.

6. Jerry Harbour, PhD, "Project Plan: Enabling Technologies for Unmanned Protection Systems," Department of Defense, Office of the Undersecretary of Defense for Acquisition, Technology \& Logistics, Joint Robotics Program, dated December 15, 2004.

7. Julie L. Marble, PhD., David J. Bruemmer, Douglas A. Few, and Don Duddenhoeffer, "Challenges in the Design and Conduct of Usability Testing of Human-Robot Control Architectures", $10^{\text {th }}$ Robotics and Remote Systems Mtg. Proceedings, pp. 458-465, ANS, Gainesville, Florida, March 28-31, 2004.

8. Sandia National Laboratories Intelligent Systems and Robotics Center. Available online at http://www.sandia.gov/isrc/Roboticvehicles.html.

9. R.J. Anderson, "SMART: A Modular Control Architecture for Robotics and Teleoperation," IEEE Robotics and Automation Society Magazine, pp. 10-18, Sept. 1995. 
10. J.T. Feddema, C. Lewis, and D.A. Schoenwald, "Decentralized Control of Cooperative Robotic Vehicles: Theory and Application," IEEE Transactions on Robotics and Automation, Vol. 18, No. 5, pp. 852-864, October 2002.

11. J. T. Feddema, R. D. Robinett, and R. H. Byrne, "An Optimization Approach to Distributed Controls of Multiple Robot Vehicles," Workshop on Control and Cooperation of Intelligent Miniature Robots, IEEE/RSJ International Conference on Intelligent Robots and Systems, Las Vegas, Nevada, October 31, 2003.

12. M.H. van Fosson, S. Fish, "Role of robotics in ground combat of the future - UGCV, PerceptOR and FCS", Proceedings of the SPIE - Unmanned Ground Vehicle Technology III, vol. 4364, p. 323-327, Orlando, FL, 2001.

13. P.S. Schenker, T. L. Huntsberger, P. Pirjanian, E. Baumgartner, E. Tunstel, "Planetary Rover Developments Supporting Mars Exploration, Sample Return and Future Human-Robotic Colonization", Autonomous Robots, vol. 14, no. 2/3, March/May 2003.

14. Stewart J. Moorehead, "Semi-Autonomous Control of the GOAT Robot Using Levels of Autonomy", $10^{\text {th }}$

Robotics and Remote Systems Mtg. Proceedings, pp. 264-269, ANS, Gainesville, Florida, March 28-31, 2004. 